\title{
ANALYTICAL AND COMPUTATIONAL ESTIMATION OF PATELLOFEMORAL FORCES IN THE KNEE UNDER SQUATTING AND ISOMETRIC MOTION
}

\author{
G. Fekete ${ }^{1}$, B. Málnási Csizmadia ${ }^{2}$, M. A. Wahab ${ }^{1}$, P. De Baets ${ }^{1}$ \\ ${ }^{1}$ Laboratory Soete, Ghent University, Belgium. \\ ${ }^{2}$ Institute of Mechanics and Machinery, Szent István University, Hungary.
}

\begin{abstract}
This study presents an intermediate step in prosthesis design, by introducing a newly developed two-dimensional mathematical, and a three-dimensional computational knee model. The analytical model is derived from Newton's law with respect to the equilibrium equations, thus based on theoretical assumptions, and experimentally obtained parameter. The numeric model is built from an existing prosthesis, involving three parts as patella, femur and tibia, and currently it is under development. The models are capable to predict - with their standard deviation - the patellofemoral (numerically tibiofemoral as well) forces in the knee joint during squatting motion. The reason why the squatting is investigated is due to its relative simplicity and the fact, that during the movement the forces reach extremity in the knee joint. The obtained forces - as a function of flexion angle - are used firstly as fundaments to the knee design method, and secondly to extend the results related to the existing isometric kinetics, where one of the newly obtained functions appears as an essential - and so far missing - input function. Most results are compared and validated to the ones found in the relevant literature and put into a dimensionless form in order to have more general meaning.
\end{abstract}

Keywords: analytical squatting model, patellofemoral forces, isometric motion

\section{INTRODUCTION}

The widespread occurrence of the various types of arthritis does not only result in significant loss in manpower, but often means immeasurable pain and suffering to many patients. Due to the limited understanding of the phenomena of the knee joint movement, the clinicians only have surgical solution, which mostly involves prosthetic replacement arthroplasty. To develop and design better prosthetic elements, several knee models - patellofemoral and tibiofemoral - have been already developed in order to investigate axial joint contact forces [25], contact points as a function of flexion [28], and the force distribution [16, 9, 17]. By knowing the acting forces in the tendons and ligaments connected to the knee joint, the development of the actual design of the prosthesis can be evaluated sooner, easier and more economically. The substantial difficulty during the building of a theoretical knee joint model, that the complexity of the joint mostly requires computational support, special programs, which does not ease the prosthetic design. But most of all, the greatest problem during the design, is the definition of the loads. This paper is an intermediate step in our research. The major aim is to develop useful methods and models for prosthesis design. The examined motion throughout our research is manly the squatting and additionally the isometric exercises. Although the squatting is considered as an everyday movement, still not that frequently used as the gait or the running. The reason we chose to investigate this specific movement is based on its relative simplicity and the fact, that during the movement the forces in the knee reach extremity. As an engineering rule of thumb, it is always recommended to design on the upper extremity of the forces. However, designing prosthesis is far from easy, thus our program is divided into segments, which are tightly interconnected. The research program involves the following main steps:

A. Creation of a 2D mathematical model to investigate the forces in the connecting ligaments of the knee joint during squatting and isometric motion. The formulas related to the isometric motion are obtained by the use of the references, while the formulas related to the squatting motion are derived from the 2D model. These forces are considered as 'external forces', and experimental validation is required.

B. Creation of a 3D computational model in MSC.ADAMS, to investigate the forces between the connecting surfaces of the femur, tibia and patella. These forces are considered as 'internal forces', and the point or area of application has to be calculated as well. 
In this paper two novel - analytic and computational - models are presented. These models are able to provide estimation - based on analytic and numeric approaches - about the external and internal forces of the knee such as the quadriceps force $\left(\boldsymbol{F}_{q}\right)$, the patellar ligament force $\left(\boldsymbol{F}_{p t}\right)$, the patello-femoral compression force between the patella and femur $\left(\boldsymbol{F}_{p}\right)$ and the tibio-femoral force $\left(\boldsymbol{F}_{t}\right)$ by deriving them as a function of knee flexion angle, mass of the person, and some 'human-bond' quantities [5]. The results are compared to the ones found in the references and to experiments as well. This study is limited to the first and partly the second step of our research program.

\section{METHODS}

\subsection{Mathematical model of squatting}

The following simplifications were considered related to the analytical model:

- The model is quasi-static,

- Two-dimensional,

- The inertial forces during the movement are neglected,

- No internal forces between the surfaces are considered,

- Only the squatting movement can be investigated with the model,

- The load is derived from the mass of the person.

Our model consisted of equilibrium equations which describe the condition of the forces connected to the femur, tibia and patella. Let us introduce the analytical model in two positions - stand and squat - and investigate the motion. It is seen that the patella rotates about a $B$ point and so does the tibia (see Fig. 1).

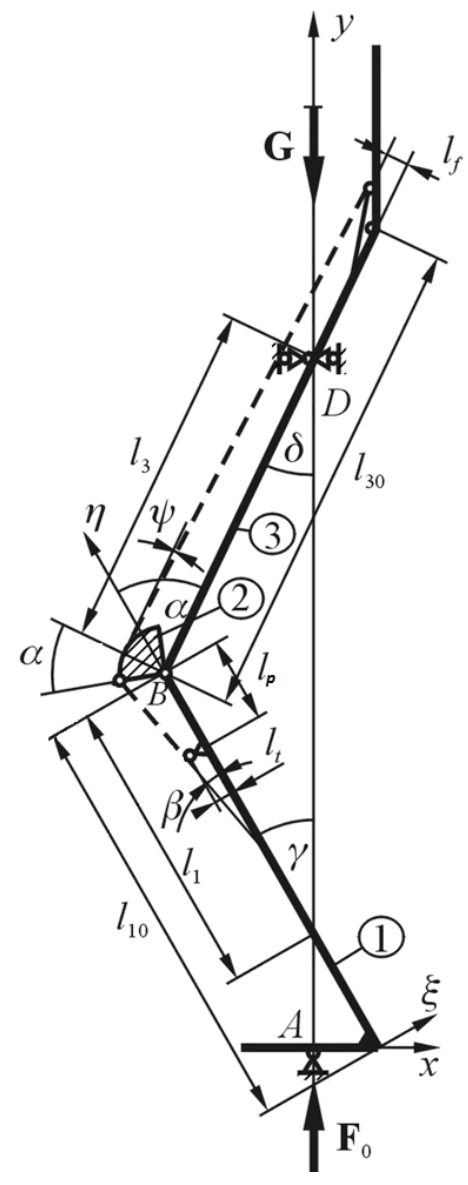

Fig. 1: Theoretical lengths of the knee joint

The model is represented at an arbitrary angle $\alpha$, and the $\boldsymbol{G}$ force is derived from the mass. The line of action of $\boldsymbol{G}$ intersects with the theoretical line of femur and tibia. The $\boldsymbol{G}$ force was chosen to be $785 \mathrm{~N}$, which equals to a person with $80 \mathrm{~kg}$ of mass but it can be arbitrarily varied. Let us fix the $y$ component of the coordinate system to this line of action, while the origin is at the ground. 
Rigid bodies will represent the femur (3), the patella (2), and the foot with the tibia (1) as well. The three rigid bodies are attached to the ground by a hinge of one degree of freedom (point $A$ ), and a roller at the intersection of the line of action and the femur (point $D$ ). At point $A$, the ground reaction force is represented as $\boldsymbol{F}_{\boldsymbol{0}}$ force, which equals to $\boldsymbol{G}$. The rigid bodies are attached to each other by strings (the elastic elongation is neglected). The calculation is presented as a function of $\alpha$, and illustrated in three different positions (Fig. 2).
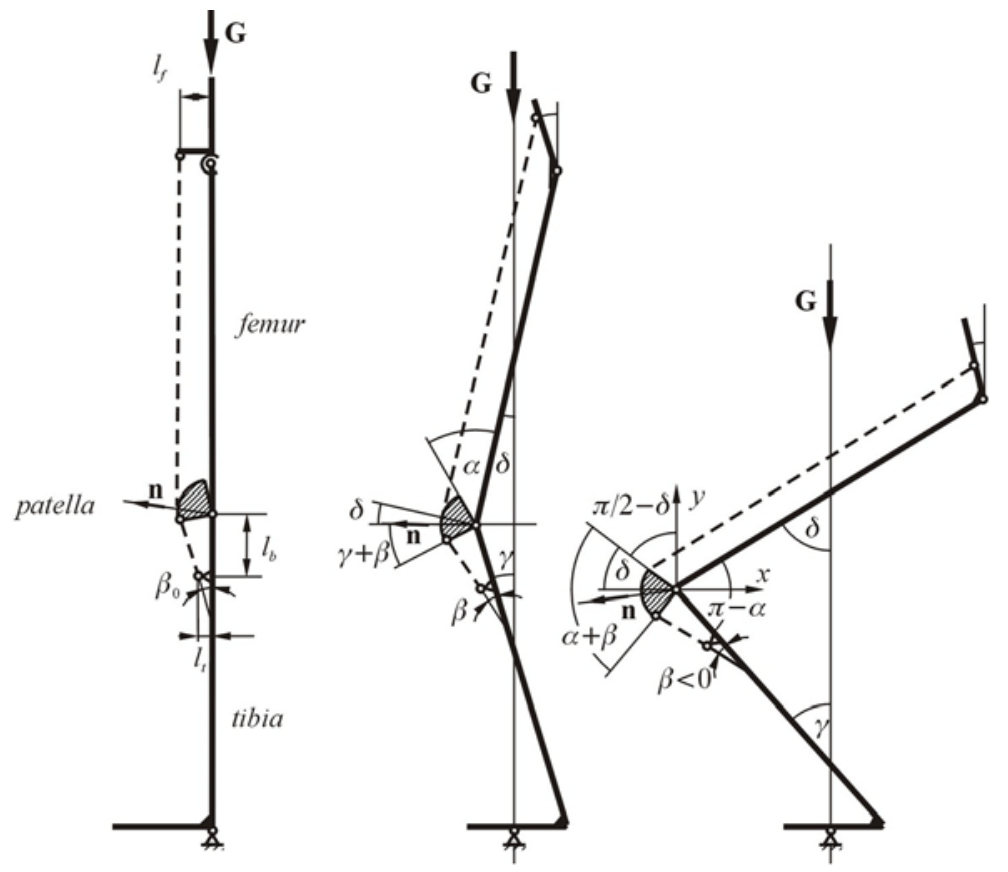

Fig. 2: 2D analytical model in three positions

The denotation of the geometric lengths in standing position:

- $I_{10}$ : the theoretical length of the tibia,

- $I_{30}$ : the theoretical length of the femur,

- $I_{p}$ : the theoretical length of the patellar tendon,

- $l_{t}$ : the perpendicular theoretical length between the axis of tibia and the surface of the patella,

- $l_{f}$ : the theoretical length between the axis of femur and the line of action of the resultant quadriceps force,

In squatting position:

- $I_{1}(\alpha)$ : the theoretical intersected length of the axis of tibia and the instantaneous line of action of the mass. Measured from the knee joint,

- $I_{3}(\alpha)$ : the theoretical intersected length of the axis of femur and the instantaneous line of action of the mass. Measured from the knee joint,

- $\alpha$ : the angle between the tibia and the femur,

- $\beta(\alpha)$ : the angle between the axis of tibia and the patellar tendon,

- $\quad \gamma(\alpha)$ : the angle between the axis of tibia and the line of action of the mass,

- $\delta(\alpha)$ : the angle between the axis of femur and the line of action of the mass, $\delta=\alpha-\gamma$,

- $\varphi(\alpha)$ : the angle between the axis of tibia and the resultant force of the upper condyles,

- $\psi(\alpha)$ : the angle between the resultant quadriceps force vector and the axis of femur.

The three elements are plotted as free-body diagrams, where the forces, angles, and the different lengths are shown on Figure 3a-d. 


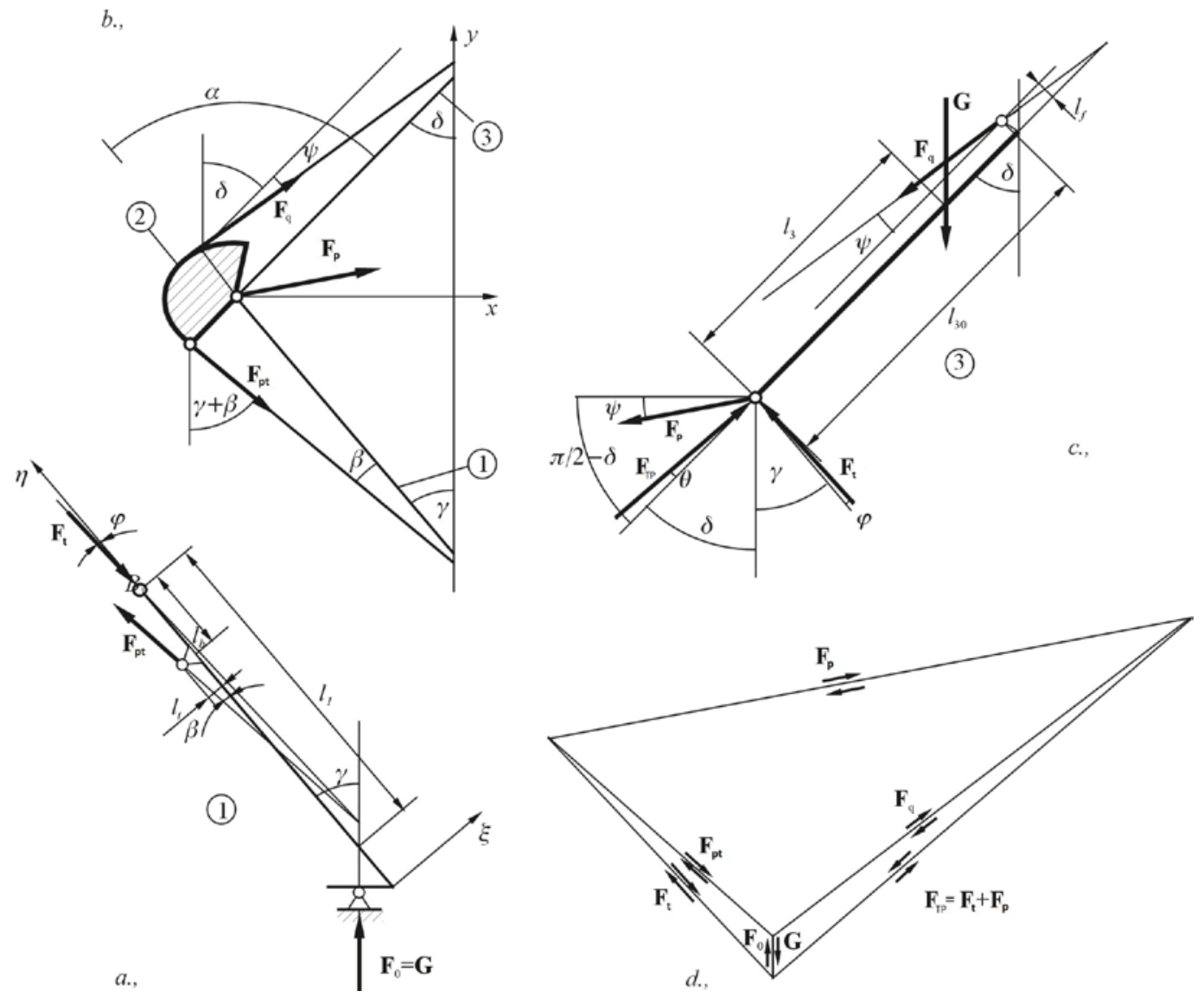

Fig. 3: The construction of the force vectors in an arbitrary position

Where the forces are:

- $F_{q}(\alpha)$ : quadriceps force,

- $\boldsymbol{F}_{p}(\boldsymbol{\alpha})$ : patellofemoral compression force,

- $F_{p t}(\alpha):$ patellar ligament force,

- $\boldsymbol{F}_{t}(\boldsymbol{\alpha})$ : tibial compression force.

The calculation is carried out by the use of the equilibrium equations as a function of angle $\alpha$.

The moment equation applied about the $z$ axis through point $B$ on the $1^{\text {st }}$ element (see Fig. 3a):

$$
\sum M_{B 1 z}=0=-l_{p} \cdot F_{p t} \cdot \sin \beta(\alpha)-l_{t} \cdot F_{p t} \cdot \cos \beta(\alpha)+l_{1}(\alpha) \cdot G \cdot \sin \gamma(\alpha)
$$

Thus the patellar force can be derived as:

$$
F_{p t}(\alpha)=G \cdot \frac{l_{1}(\alpha) \cdot \sin \gamma(\alpha)}{l_{p} \cdot \sin \beta(\alpha)+l_{t} \cdot \cos \beta(\alpha)}
$$

In order to simplify and generalize the results let us to introduce dimensionless geometric quantities and forces, thus the acting force will be calculated in a normalized form with respect to the force derived from the body mass $(\boldsymbol{G})$.

- $\quad \lambda_{1}(\alpha)=l_{1}(\alpha) / l_{10}$ : dimensionless, intersected tibia length function,

- $\quad \lambda_{3}(\alpha)=l_{3}(\alpha) / l_{30}$ : dimensionless, intersected femur length function,

- $\lambda_{p}=l_{p} / l_{10}$ : dimensionless length of patellar tendon,

- $\lambda_{t}=l_{t} / l_{10}$ : dimensionless thickness of shin,

- $\lambda_{f}=l_{f} / l_{30}$ : dimensionless thickness of thigh. 
By the introduction of these quantities, the acting force in the patellar ligament is:

$$
\frac{F_{p t}(\alpha)}{G}=\frac{\lambda_{1}(\alpha) \cdot \sin \gamma(\alpha)}{\lambda_{p} \cdot \sin \beta(\alpha)+\lambda_{t} \cdot \cos \beta(\alpha)}
$$

The scalar equilibrium equations related to the $1^{\text {st }}$ element in the $\xi$ - $\eta$ coordinate system (see Fig. 3a):

$$
\begin{gathered}
\sum F_{i \eta}=0=-F_{t} \cdot \cos \varphi(\alpha)+F_{p t} \cdot \cos \beta(\alpha)+G \cdot \cos \gamma(\alpha) \\
\sum F_{i \xi}=0=F_{t} \cdot \sin \varphi(\alpha)-F_{p t} \cdot \sin \beta(\alpha)+G \cdot \sin \gamma(\alpha)
\end{gathered}
$$

By some simplification the angle between the axis of tibia and the resultant force of the upper condyles can be derived from Eq. (4) and Eq. (5) as:

$$
\varphi(\alpha)=\operatorname{arctg}\left[\frac{\left(\lambda_{1}(\alpha)-\lambda_{p}\right) \cdot \operatorname{tg} \beta(\alpha)-\lambda_{t}}{\lambda_{1}(\alpha) \cdot \operatorname{tg} \gamma(\alpha)+\lambda_{p} \cdot \operatorname{tg} \beta(\alpha)+\lambda_{t}} \cdot \operatorname{tg} \gamma(\alpha)\right]
$$

By the use of angle $\varphi$ the acting normal force in the tibia can be derived from Eq. (4) or Eq. (5) as well:

$$
\frac{F_{t}(\alpha)}{G}=\frac{F_{p t}}{G} \cdot \frac{\cos \beta(\alpha)}{\cos \varphi(\alpha)}+\frac{\cos \gamma(\alpha)}{\cos \varphi(\alpha)}
$$

The moment equation applied about the $z$ axis through point $B$ on the $3^{\text {rd }}$ element (see Fig. 3c):

$$
\sum M_{i b 3}=0=l_{f} \cdot F_{q} \cdot \cos \psi(\alpha)+l_{30} \cdot F_{q} \cdot \sin \psi(\alpha)-l_{3}(\alpha) \cdot G \cdot \sin \delta(\alpha)
$$

Taking into account that $\delta=\alpha-\gamma$, and assuming $\psi=0$, the quadriceps force in the tendon:

$$
\frac{F_{q}(\alpha)}{G}=\frac{\lambda_{3}(\alpha) \cdot \sin (\alpha-\gamma(\alpha))}{\lambda_{f}}
$$

The $\psi=0$ assumption means that the direction of the resultant, acting forces in the quadriceps muscle are parallel with the axis of femur, since the muscle is connected to under the hip bone and stretches out until the frontal surface (facies patellaris) [26], thus this approximation is acceptable. The scalar equilibrium equations related to the $2^{\text {nd }}$ element in the $x-y$ coordinate system (see Fig. $3 b$ ):

$$
\begin{aligned}
& \sum F_{i x}=0=F_{q}(\alpha) \cdot \sin \delta(\alpha)+F_{t}(\alpha) \cdot \sin (\gamma(\alpha)+\beta(\alpha))+F_{p x} \\
& \sum F_{i y}=0=F_{q}(\alpha) \cdot \cos \delta(\alpha)-F_{t}(\alpha) \cdot \cos (\gamma(\alpha)+\beta(\alpha))+F_{p y}
\end{aligned}
$$

Out of Eq. (10) and Eq. (11) equations the magnitude of the patellar compression force can be derived by the use of $x, y$ coordinates with respect to the body mass force:

$$
\frac{F_{p}(\alpha)}{G}=\frac{\sqrt{F_{p x}^{2}+F_{p y}^{2}}}{G}=\frac{\sqrt{F_{q}(\alpha)^{2}+F_{t}(\alpha)^{2}-2 \cdot F_{q}(\alpha) \cdot F_{t}(\alpha) \cdot \cos (\beta(\alpha)+\delta(\alpha)+\gamma(\alpha))}}{G}
$$

Since all the forces are mathematically described, by the use of $\lambda_{1}(\alpha), \lambda_{3}(\alpha), \lambda_{b}, \lambda_{t}, \lambda_{f}, \beta(\alpha), \gamma(\alpha), \delta(\alpha), \varphi(\alpha)$ dimensionless functions and constants [5] with - their standard deviation - the formulas of the forces are complete and usable to estimate the forces in the knee joint during squatting.

\subsection{Mathematical model of isometric motions}

Several authors investigated the ratio of patellofemoral forces under isometric extension and flexion exercises in the relevant literature $[15,17,7,1,8]$ with very similar results. In case of isometric motion investigation, it is always emphasized that generally the applied loads are relatively small (e.g. approx. $50 \mathrm{~N}$ [27] or $2.2 \mathrm{Nm}$ derived from $0.5 \mathrm{~kg}$ of weight [17]) and correlations between the forces are always described as a ratio $\left(\boldsymbol{F}_{p t} / \boldsymbol{F}_{\boldsymbol{q}}, \boldsymbol{F}_{p} / \boldsymbol{F}_{\boldsymbol{q}}\right)$ in the function of flexion angle $\alpha$.

This approach only shows the ratio, but does not describe the forces individually. In order to gain more information about the forces, the quadriceps force has to be determined as well. The function of the quadriceps force is mostly investigated experimentally [22] or semi-experimentally by using theoretical 
assumptions and experimental methods to investigate the internal forces [30]. In the experimental work they took into account 'human-bond' quantities such as muscle force-length factor as a function of knee and hip flexion angle, and physiological cross sectional area of the muscle. These quantities are not easy to be measured.

The most known analytical descriptions among all are originated to Van Eijden's and Yamaguchi's models $[27,29]$, where the authors constructed a 2D model, acting in the sagittal plane. The elements were considered as rigid bodies and during the movement the femur was fixed while the tibia was rolling-sliding on the condyle of the femur. Model operates the following way: an $\boldsymbol{F}_{\boldsymbol{q}}$ force is exerted by the quadriceps which is balanced by the $\boldsymbol{F}_{\boldsymbol{p} t}$ patellar ligament force and the $\boldsymbol{F}_{\boldsymbol{p}}$ patellar compression force during the motion. Gravitational forces are not taken into account in this kind of investigation.

The ratio of the patellofemoral forces determined by various authors are summarized and shown in Figure 4 and 5.

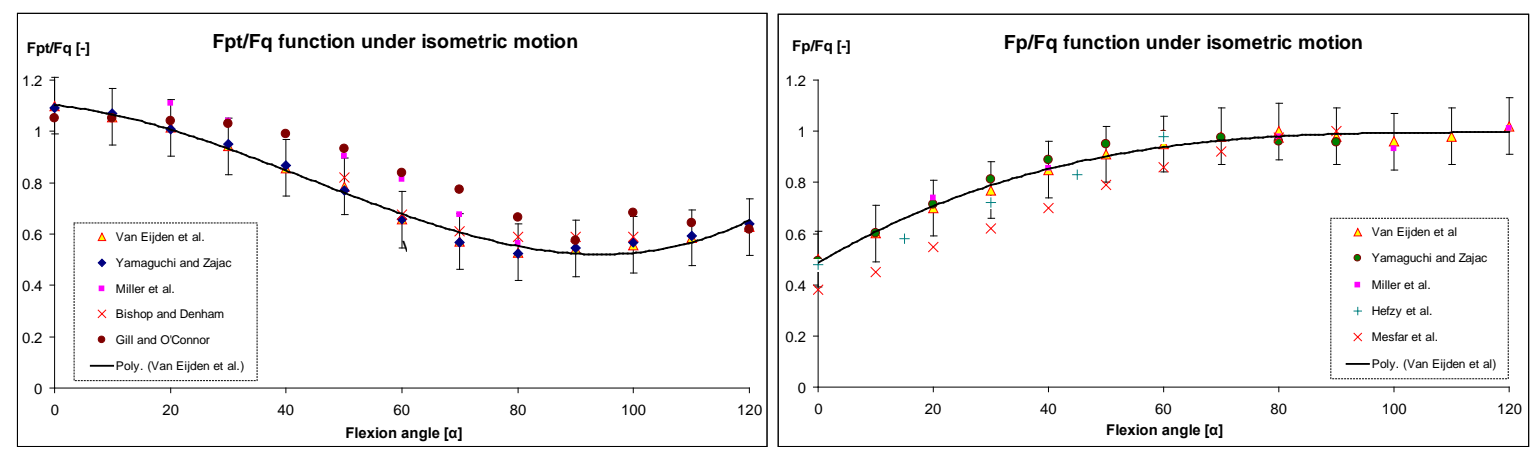

Fig. 4 and 5: Fpt/Fq and Fp/Fq relationship by Van Eijden et al.

As it is seen, the results of the authors are in good agreement and in addition, Van Eijden's model includes the error as well. Since the experimental and numerical results obtained by the other authors are mostly in the range of the error, it is adequate to use further on Van Eijden's mathematical model.

The cubic approximate functions of Van Eijden's model with standard deviation and 1.1\% error of fitting are:

$$
\begin{aligned}
& \frac{F_{p t}}{F_{q}}(\alpha)=1.1383 \cdot 10^{-6} \cdot \alpha^{3}-1.4934 \cdot 10^{-4} \cdot \alpha^{2}-2.20944 \cdot 10^{-3} \cdot \alpha+1.1024 \pm S D_{F_{p t} / F_{q}} \\
& \frac{F_{p}}{F_{q}}(\alpha)=3.3508 \cdot 10^{-7} \cdot \alpha^{3}-1.1516 \cdot 10^{-4} \cdot \alpha^{2}+13.2417 \cdot 10^{-3} \cdot \alpha+0.486 \pm S D_{F_{p} / F_{q}}
\end{aligned}
$$

Where $S D_{F_{p} t / F_{q}}=0.1$ and $S D_{F_{p} / F_{q}}=0.11$.

The $\boldsymbol{F}_{p} / \boldsymbol{F}_{q}(\alpha)$ and $\boldsymbol{F}_{p t} / \boldsymbol{F}_{\boldsymbol{q}}(\alpha)$ functions depend on the angle of flexion, but the patellar compression force and the patellar ligament force cannot be directly calculated since the $\boldsymbol{F}_{\boldsymbol{q}}$ force is not given either.

The $\boldsymbol{F}_{\boldsymbol{q}}$ force always appears as a known, externally applied force, and so far, no analytic function was published about its calculation. In section 2.1, Eq. (9) gives an analytic solution to $\boldsymbol{F}_{\boldsymbol{q}}(\alpha)$ which function only depends on the angle $\alpha$. With this unique approach the other patellofemoral forces can be calculated as well, by simply multiplying $\boldsymbol{F}_{p} / \boldsymbol{F}_{q}(\alpha)$ and $\boldsymbol{F}_{p t} / \boldsymbol{F}_{q}(\alpha)$ functions with $\boldsymbol{F}_{\boldsymbol{q}}(\alpha)$ function, thus obtaining $\boldsymbol{F}_{p t}(\alpha)$ and $\boldsymbol{F}_{\boldsymbol{p}}(\alpha)$ individually. Let us define $\boldsymbol{F}_{\boldsymbol{p t}}(\alpha)$ and $\boldsymbol{F}_{\boldsymbol{p}}(\alpha)$ functions as:

$$
\begin{gathered}
F_{p t}=\frac{F_{p t}}{F_{q}}(\alpha) \pm S D_{F_{p t} / F_{q}} \cdot F_{q}(\alpha) \pm S D_{F_{q}}(\alpha) \\
F_{p}=\frac{F_{p}}{F_{q}}(\alpha) \pm S D_{F_{p} / F_{q}} \cdot F_{q}(\alpha) \pm S D_{F_{q}}(\alpha)
\end{gathered}
$$

By simply multiplying the two functions the demanded forces can be obtained. Nevertheless, the two functions have different standard deviation (SD) which has to be unified and calculated.

The standard deviation of $\boldsymbol{F}_{q}(\alpha)$ depends on the $\lambda_{3}(\alpha)$ function, which can be taken from the references [5]:

$$
\lambda_{3}(\alpha)=-0.0022 \cdot \alpha+0.86 \pm S D_{\lambda 3}(\alpha)
$$


Where,

$$
S D_{\lambda 3}=t \cdot S_{\lambda 3}(\alpha)=1.57 \cdot 10^{-6} \cdot \alpha^{2}+5.4 \cdot 10^{-4} \cdot \alpha+0.057
$$

is standard deviation function of $\lambda_{3}(\alpha)$, and $t=1.96$ according to the $95 \%$ confidence.

Substituting Eq. (17) and Eq. (18) into Eq. (9), the standard deviation of $\boldsymbol{F}_{\boldsymbol{q}}(\alpha)$ force function is obtained as follows:

$$
S D_{F_{q}}(\alpha)=-0.0047 \cdot \alpha^{2}+4.1298 \cdot \alpha-7.1692
$$

Since the functions are available, the standard deviation must be also determined for $\boldsymbol{F}_{p t}(\alpha)$ and $\boldsymbol{F}_{p}(\alpha)$. To determine the global standard deviation for these functions, let us consider an $f(x)$ function with e.g. three parameters as $p_{1}, p_{2}, p_{3}$. Each parameter has a measured standard deviation denoted as $S D_{p 1}, S D_{p 2}$, and $S D_{p 3}$. The global deviation of the function can be derived from the principle of error propagation $[11,24]$ as follows:

$$
S D_{f}=\left|\frac{\partial f}{\partial p_{1}}\right| \cdot S D_{p_{1}}+\left|\frac{\partial f}{\partial p_{2}}\right| \cdot S D_{p_{2}}\left|\frac{\partial f}{\partial p_{3}}\right| \cdot S D_{p_{3}}
$$

Expanding the principle to the case of multiple functions with one variable, let us consider the force functions in Eq. (15) and Eq. (16):

$$
\begin{gathered}
S D_{F_{p t}}=\left|\frac{\partial F_{p t}}{\partial F_{q}}\right| \cdot S D_{F_{q}}(\alpha)+\left|\frac{\partial F_{p t}}{\partial F_{p t} / F_{q}}\right| \cdot S D_{F_{p t} / F_{q}} \\
S D_{F_{p}}=\left|\frac{\partial F_{p}}{\partial F_{q}}\right| \cdot S D_{F_{q}}(\alpha)+\left|\frac{\partial F_{p}}{\partial F_{p} / F_{q}}\right| \cdot S D_{F_{p} / F_{q}}
\end{gathered}
$$

By undertaking the partial differentiation and the multiplication, then simplifying the equations the following results are obtained related to the standard deviation:

$$
\begin{gathered}
S D_{F_{p t}}=1.1383 \cdot 10^{-6} \cdot\left(1.57 \cdot 10^{-6} \cdot \alpha^{2}+5.4 \cdot 10^{-4} \cdot \alpha+0.057\right) . \\
\sqrt{(73.6579+\alpha)^{2} \cdot\left(13148.1-204.854 \cdot \alpha+\alpha^{2}\right)^{2}}+\sqrt{\frac{G^{2} \cdot \lambda_{3}(\alpha)^{2} \cdot \sin (\alpha-\gamma(\alpha))^{2}}{\lambda_{f}}} \cdot 0.1 \\
S D_{F_{p}}=3.3508 \cdot 10^{-7} \cdot\left(1.57 \cdot 10^{-6} \cdot \alpha^{2}+5.4 \cdot 10^{-4} \cdot \alpha+0.057\right) \cdot \\
\sqrt{(28.8539+\alpha)^{2} \cdot\left(50267.1-372.533 \cdot \alpha+\alpha^{2}\right)^{2}}+\sqrt{\frac{G^{2} \cdot \lambda_{3}(\alpha)^{2} \cdot \sin (\alpha-\gamma(\alpha))^{2}}{\lambda_{f}}} \cdot 0.11
\end{gathered}
$$

Thus the global standard deviations of the functions are also obtained. The results are general with valid deviation and suitable to calculate directly the forces as a function of flexion angle $\alpha$.

\subsection{Computational squatting model}

\subsubsection{Knee joint geometry}

The model was built in the MSC.ADAMS program system (see Fig. 6 and 7). The bones, such as the tibia, patella and femur were assumed as rigid bodies, since the influence of deformation in this study is irrelevant. The geometry of the femur and tibia is based on a prosthesis prototype, which is under tests and developments. 

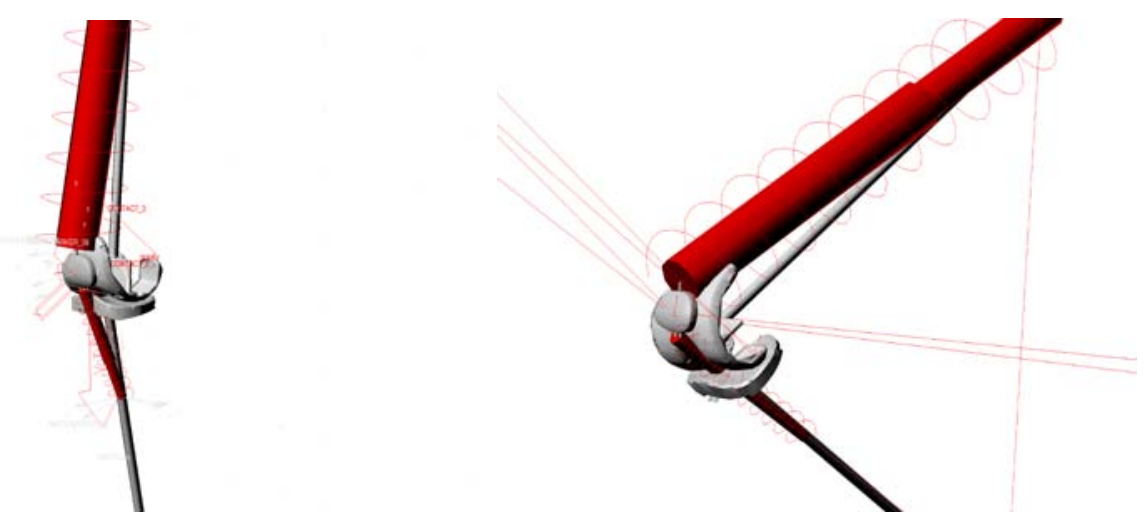

Fig. 6 and 7: MSC.ADAMS knee model in two positions

\subsubsection{Ligaments, muscles}

Only the patellar ligament and the quadriceps muscle were considered in the computational model. The quadriceps muscle and the patellar ligament were modelled as simple linear springs. The stiffness coefficient was set to $130 \mathrm{~N} / \mathrm{mm}$ and the damping coefficient to $0.15 \mathrm{Ns} / \mathrm{mm}$ in case of both springs. That corresponds with the measured values in the literature $[12,18]$.

\subsubsection{Loads, constraints and boundary conditions}

The same load ( $\boldsymbol{G}=785 \mathrm{~N}$ ) was applied in case of the computational model as it was in the analytical model (see Fig. 6). The point of application was the femur distalis. The femur distalis was constrained by a GENERAL POINT MOTION, where all the coordinates can be prescribed. Only one prescription was set: the endpoint of the femur (distalis) can only perform translational motion along the $y$ axis.

The ankle part of the model was constrained by a SPHERICAL JOINT, which allows rotation about all axes, but all the translational motions equal to zero in that point. By applying this constraint, the tibia can perform a natural rotation, and later on, it can be kinematically analyzed.

Between the femur, tibia and patella, CONTACT constraints were set according to Coulomb's law with respect to the very low friction $\left(\mu_{s}=0.0003 \mu_{d}=0.0001\right)$ in case of real joints $[19,21]$. The relationship between the internal (contact forces) and external forces was not analyzed in this study.

\subsubsection{Solver, simulation, post-procession}

The MSC.ADAMS creates and solves simultaneously linear or non-linear Ordinary Differential Equations (ODE) and non-linear Differential-Algebraic Equations (DAE). The DAE is distinct from ODE since it involves an unknown function with its derivatives. Briefly the solution of any DAE is the following: firstly, a consistent initial value must be found, and secondly the trajectory of the DAE must be computed.

The simulation time was set to $0.045 \mathrm{sec}$ in 200 time steps. GSTIFF [6] integrator was used for integrating the ODE and DAE of the motion. The solver routine was set to work maximum 1e-003 tolerance of error, while the maximum order of the polynomial was defined to 12 . The solution converged very quickly with these parameters.

The post-procession was carried out in the ADAMS and partly in the Excel [13]. The ADAMS could compute the directly the forces, velocities and accelerations, but not the rotations. The flexion angle was derived by integrating the angular velocities of the femur and tibia about the $x$ axis, taking into account that the model was in an initial 15 degree of squat in the beginning of the motion. In order to deal with the fact that the motion is three-dimensional the angles are decomposed to three separate angles. These angles are Euler angles, thus an order was chosen (313) to define them during the calculation. After integrating the appropriate angular velocities the results were summarized in charts by using the Excel program. 


\section{RESULTS AND DISCUSSION}

In Fig. 8-9-10-11-12-13 the relationship between the angle of flexion and the calculated forces are expressed in case of squatting motion as a function of flexion angle.
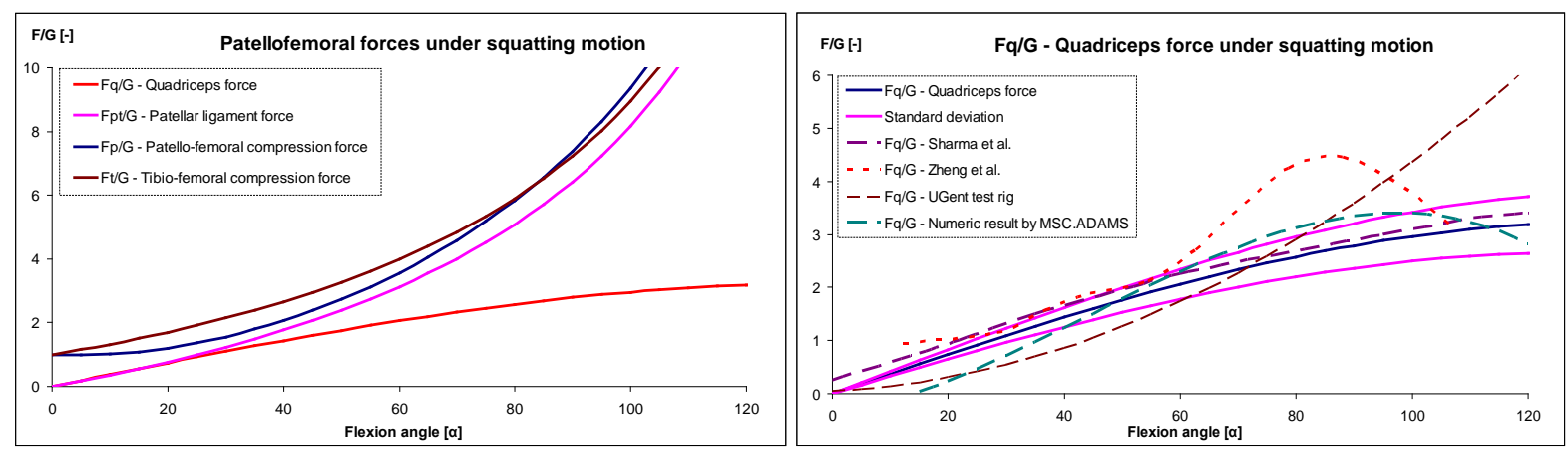

Fig. 8 and 9: Patellofemoral forces and the quadriceps force under squatting motion
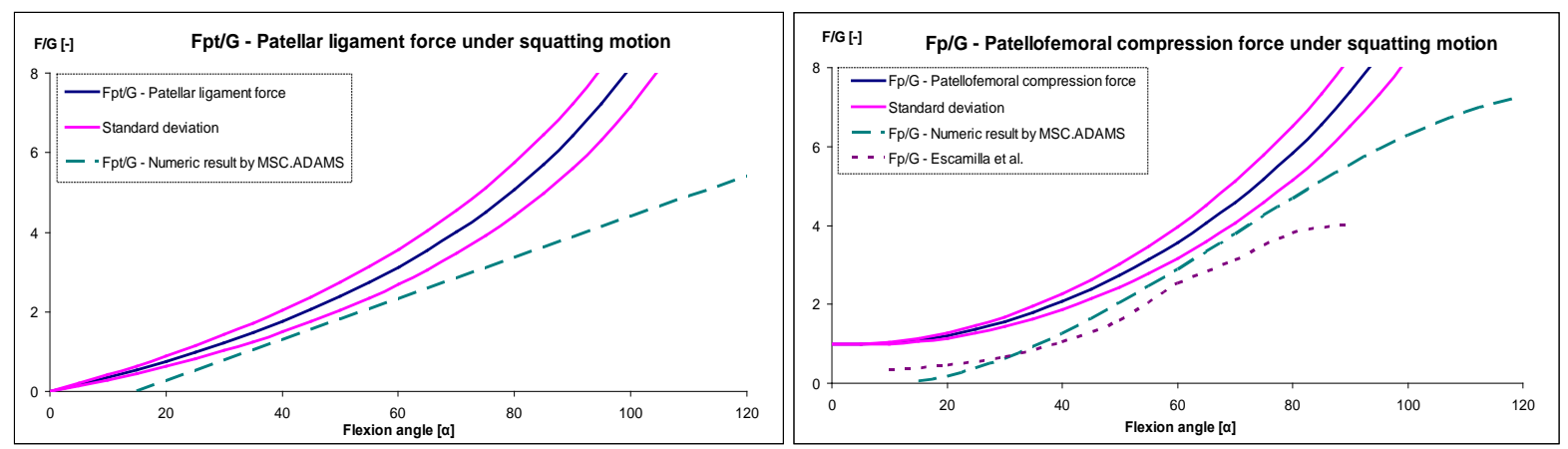

Fig. 10 and 11: Patellar ligament and Patellofemoral force under squatting motion
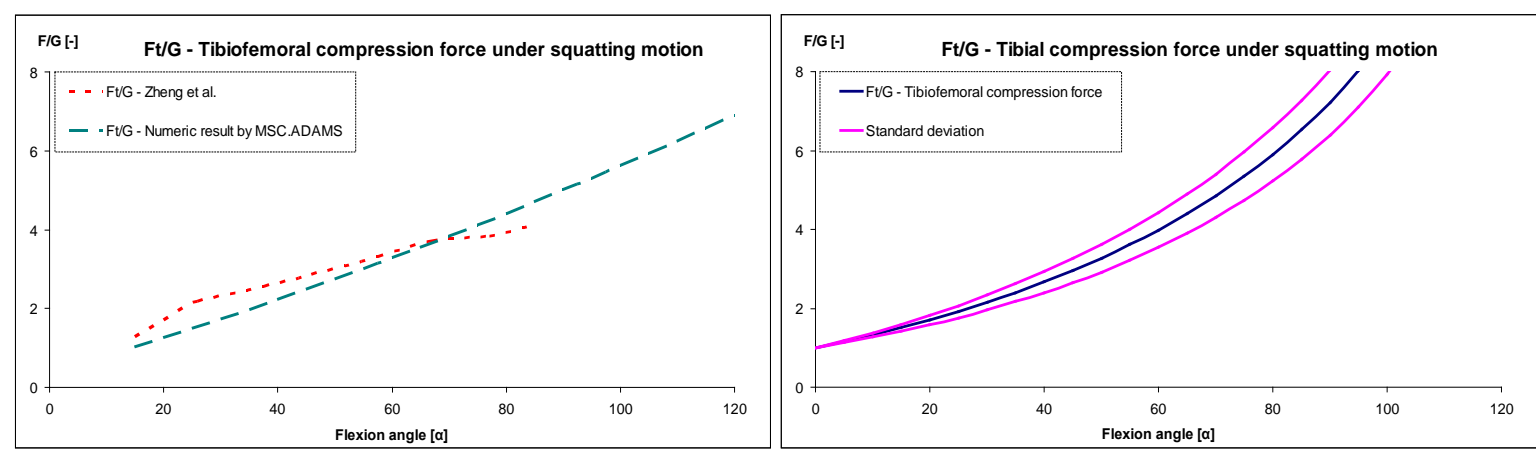

Fig. 12 and 13: Tibiofemoral and Tibial compression force under squatting motion

In Figure 8, all the four patellofemoral forces are summarized in one diagram, where the patellar-ligament, patellofemoral compression and the tibiofemoral compression forces have rapidly growing tendency as a function of flexion angle, while the quadriceps force reaches its peak value approximately at 120 degree (see in Fig. 9). The quadriceps force has a good accordance with another author's result [22], who used reversed dynamics approach based on in vivo measurement of knee kinematics. Beside Sharma's result, experiments were carried out at the Ghent University with an Oxford type test rig. The test rig is capable to measure the quadriceps force, and the ankle forces. The experimental test provided fairly good result until 80 degree of flexion (see in Fig. 9), above that, at the maximum value of the function; the force is overpredicted approximately with the factor of two, similarly to other authors' results who used Oxford type test rig. This over-prediction is originated to the Oxford type test rig method [14], and considered average compared other authors [2, 23, 20]. The explanation of the over-prediction is the following: during the dissection of the cadaver knee, the quadriceps muscles are detached from the femur, and the knee capsule is either opened or completely removed. That results that the complete body weight $(\boldsymbol{G})$ is carried by only the quadriceps and the patellar ligament, thus the estimated - or measured - loads appear higher than the physiological.

Other authors [30] have achieved closely same result with combined (experimental work and theoretical assumptions) methods, although the peak value of the quadriceps force and the tibiofemoral force were both estimated approximately at 90 degree of flexion angle, which corresponds fairly well, if the results were obtained by Oxford type test rig $[2,23,20]$. In case the results are obtained by carrying out 
measurements and calculations on human subjects, the peak of the forces (patellofemoral, tibiofemoral forces, including the quadriceps force as well) is shifted positively approximately 30 degree of flexion angle, thus the peak of the forces are approximately between 120-130 degree of flexion angle [22, 10, 3].

In this study a three-dimensional computational model was created and simulated as well, and the results were plotted in Figure 9-10-11-12. In case of the patellofemoral compression force, the analytical and numerical results are compared to the experimental results from the related literature [4] in Figure 11. The analytical model over-predicts the experimental with a factor of one until 60 degree, and a factor of more than two beyond 80 degree of flexion angle. The numeric approach gives better approximation about the patellofemoral force since the ADAMS model has similar characteristic, and 0.4 factor of average difference compared to the results of Escamilla et al., while the analytical model does not break off between 90 and 120 degree of flexion angle, where the estimated peak point is located.

The tibiofemoral force cannot be calculated by the analytical model, thus only the ADAMS model was compared to the related references (see Fig. 10). The numeric result is in good agreement with other author's result [30], although there was no more comparable date to match above 90 degree of flexion.

Comparable literature for the tibial compression force and patellar ligament force were not found, and only the analytic patellar ligament force could be compared to the numerical results (see Fig. 10). It is clearly seen, that the characteristic of the forces are parabolic and their values are converging to infinite, thus they could be valid only at lower angles.

In Figure 14-15-16 the forces in case of isometric motion are plotted against the flexion angle.

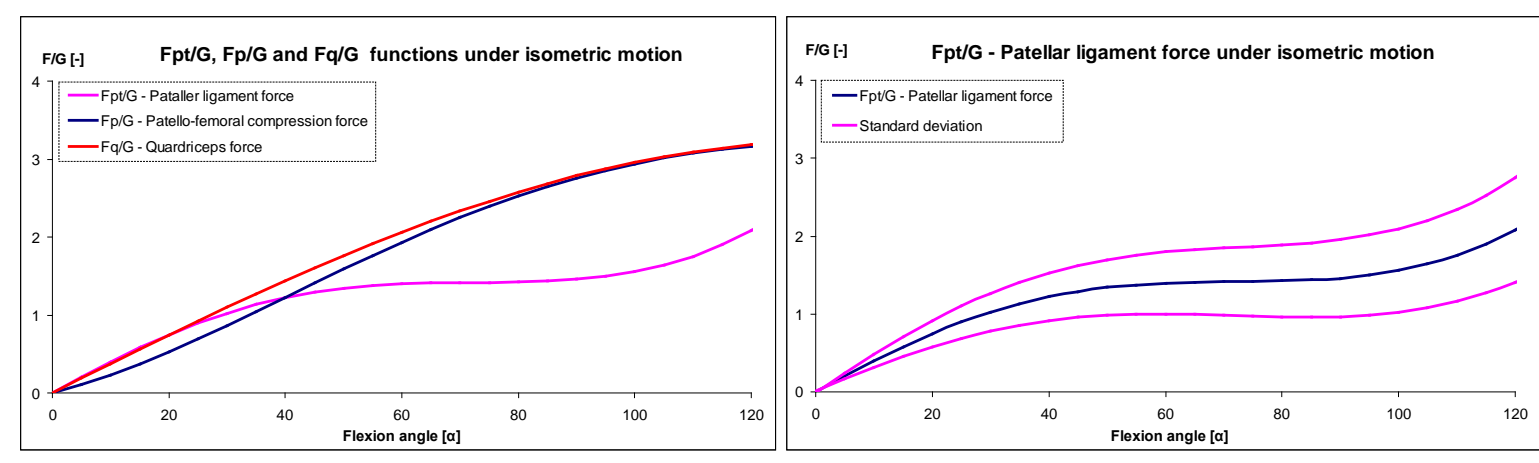

Fig. 14 and 15: Patellofemoral and Patellar ligament force under isometric motion

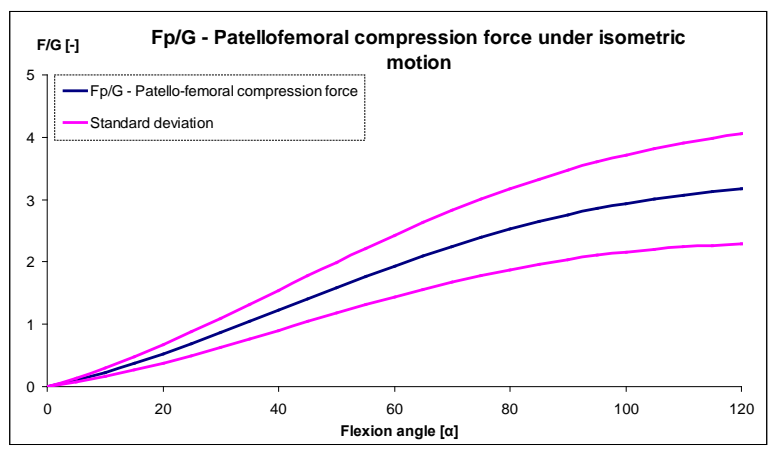

Fig. 16: Patellofemoral compression force under isometric motion

As it is seen, the forces are substantially smaller under isometric motion than during squatting. By the use of the $\boldsymbol{F}_{\boldsymbol{q}}(\alpha)$ force function, the patellofemoral force alongside with the patellar ligament forces can be calculated and plotted with their margin of error as well.

\section{CONCLUSION}

In summary, two new models were presented in this paper, an analytic and a numeric, which are capable to predict - with their standard deviation - the patellofemoral and tibiofemoral forces in the knee during squatting motion. The analytic model is based on theoretical assumptions and experimentally determined parameters based on multiple human participants, while the numeric, or so called ADAMS model, is built from an existing prosthesis, which is in prototype phase.

The results of the analytical model - especially the quadriceps force - showed good accordance with the compared experimental and analytical results taken from the references. 
The obtained $\boldsymbol{F}_{\boldsymbol{q}}(\alpha)$ force function is extended for further use as an input function for isometric motion, since all the descriptive relationships found in the references provide a ratio of the patellofemoral forces divided by the quadriceps force, thus the single components are not possible to derive.

As a further step, the analytic model will be complemented with other flexion angle dependent parameters in order to decrease the deviation between the experiemental result and the analytic, while the in case of the ADAMS model, the internal forces such as the friction and normal force between the connecting surfaces will be investigated as a function of flexion angle.

\section{ACKNOWLEDGMENT}

The study was supported by the FWO (project number: G022506), the Universiteit Gent - Labo Soete, and the Szent István University - Faculty of Mechanical Engineering and Mechanical Engineering PhD School.

\section{References}

[1.] Bishop, R.E.D., Denham, R.A. A note on the ratio between tensions in the quadriceps tendon and infrapatellar ligament. Engineering in Medicine, 6, 53-54, 1977.

[2.] Churchill, D.L., Incavo, S.J., Johnson, J.J., Beynnon, B.D. The influence of femoral rollback on patellofemoral contact loads in total knee arthroplasty. Journal of Arthroplasty, 16, 909-918. 2001.

[3.] Dahlkvist, N.J., Mayo, P., Seedhom, B.B. Force during squatting and rising during a deep squat. Engineering in Medicine, 11, 69-76, 1982.

[4.] Escamilla, R.F., Zheng, N., MacLeod, T.D., Edwards, W.B., Hreljak, A., Fleisig, G.S., Wilk, K.E., Moorman III, C.T., Imamura, R. Patellofemoral compressive force and stress during the forward and side lunges with and without stride. Clinical Biomechanics, 23, 1026-1037, 2008.

[5.] Fekete, G. Development of mathematical knee model and its validation. MSc. thesis, Ghent University, Ghent, 2007.

[6.] Gear, C.W. The Simultaneous Solution of Differential Algebraic Systems. IEEE Transactions on Circuit Theory, CT-18, No.1, 89-95, 1971.

[7.] Gill, H.S., O'Connor, J.J. Biarticulating two-dimensional computer model of the human patellofemoral joint. Clinical Biomechanics, 11, 81-89, 1996.

[8.] Hefzy, M.S., Yang, H. A three-dimensional anatomical model of the human patello-femoral joint for the determination of patello-femoral motions and contact characteristics. Journal of Biomedical Engineering, 15, 289-302, 1993.

[9.] Hsu, R.W., Himeno, S., Coventry, M.B., Chao, E.Y. Normal axial alignment of the lower extremity and load-bearing distribution at the knee. Clinical Orthopaedics and Related Research, 255, 215227, 1990.

[10.] Komistek, R.D., Kane, T.R., Mahfouz, M., Ochoa, J.A., Dennis, D.A. Knee mechanics: a review of past and present techniques to determine in vivo loads. Journal of Biomechanics, 38, 215-228, 2005.

[11.] $\mathrm{Ku}, \mathrm{H}$. Notes on the use of propagation of error formulas. Journal of research of the National Bureau of Standards - C. Engineering and Instrumentation, 70, 263-273, 1966.

[12.] Ling, Z-K., Guo, H-Q., Boersma, S. Analytical study on the kinematic and dynamic behaviors of the knee joint. Medical Engineering \& Physics, 19, 29-36, 1997.

[13.] MacDonald, M. Excel for starters: The missing manual. Pogue Press/O'Riley, Sebastopol, 2005.

[14.] Mason, J.J., Leszko, F., Johnson, T., Komistek, R.D. Patellofemoral joint forces. Journal of Biomechanics, 41, 2337-2348, 2008.

[15.] Mesfar, W., Shirazi-Adl, A. Biomechanics of the knee joint under various quadriceps forces. The Knee, 12, 424-434, 2005.

[16.] Miller, E.J., Riemer, R.F., Donahue, T.L.H., Kaufman, K.R. Experimental validation of a tibiofemoral model for analyzing joint force distribution. Journal of Biomechanics, 42, 1355-1359, 2009.

[17.] Miller, R.K., Murray, D.W., Gill, H.S., O'Connor, J.J., Goodfellow, J.W. In vitro patellofemoral joint force determined by a non-invasive technique. Clinical Biomechanics, 12, 1-7, 1997.

[18.] Momersteeg, T.J., Blankevoort, L., Huiskes, R., Kooloos, J.G., Kauer, J.M., Hendriks, J.C. The effect of variable relative insertion orientation of human knee bone-ligament-bone complexes on the tensile stiffness. Journal of Biomechanicsm, 28, 745-752, 1995.

[19.] Mow, V.C., Soslowky, L.J. Friction, lubrication \& wear of diarthrodial joints. In. Basic Orthopaedic Biomechanics. Chap. 6 (Edited by Mow, V.C. and Hayes, W.C.). Raven Press, New York, 1991.

[20.] Petersilge, W.J., Oishi, C.S., Kaufman, K.R., Irby, S. E., Colwell, C.W. The effect of trochlear design on patellofemoral shear and compressive forces in total knee arthroplasty. Clinical Orthopaedics and Related Research, 309, 124-130, 1994. 
[21.] Quian, S.H., Ge, S.R., Wang, Q.L. The frictional coefficient of bovine knee articular cartilage. Journal of Bionic Engineering, 3, 79-85, 2006.

[22.] Sharma, A., Leszko, F., Komistek, R.D., Scuderi, G.R., Cates, H.E., Liu, F. In vivo patellofemoral forces in high flexion total knee arthroplasty. Journal of Biomechanics, 41, 642-648, 2008.

[23.] Singerman, R., Berilla, J., Archdeacon, Peyser, A. In vitro forces in the normal and cruciatedeficient knee during simulated squatting motion. Journal of Biomechanical Engineering, 121, 234242, 1999.

[24.] Skoog, D., Holler, J., Crouch, S. Principles of Instrumental Analysis. $6^{\text {th }}$ Ed., Thomson Brooks/Cole Press, Belmont, 2007.

[25.] Smith, S. M., Cockburn, R.A., Hemmerich, A., Li, R.M., Wyss, U.P. Tibiofemoral joint contact forces and knee kinematics during squatting. Gait \& Posture, 27, 376-386, 2008.

[26.] Szentágothai, J. Funkcionális anatómia I. (Functional Anatomy I.). Medicina Könyvkiadó Zrt (Medicina Press), Budapest, 2006.

[27.] Van Eijden, T.M.G.J., Kouwenhoven, E., Verburg, J., Weijs, W.A. A mathematical model of the patellofemoral joint. Journal of Biomechanics, 19, 219-229, 1986.

[28.] Wretenberg, P., Ramsey, D.K., Németh, G. Tibiofemoral contact points relative to flexion angle measured with MRI. Clinical Biomechanics, 17, 477-485, 2002.

[29.] Yamaguchi, G.T., Zajac, F.E. A planar model of the knee joint to characterize the knee extensor mechanism. Journal of Biomechanics, 22, 1-10, 1989.

[30.] Zheng, N., Fleisig, G.S., Escamilla, R.F., Barrentine, S.W. An analytical model of the knee for estimation of internal forces during exercise. Journal of Biomechanics, 31, 963-967, 1998. 